
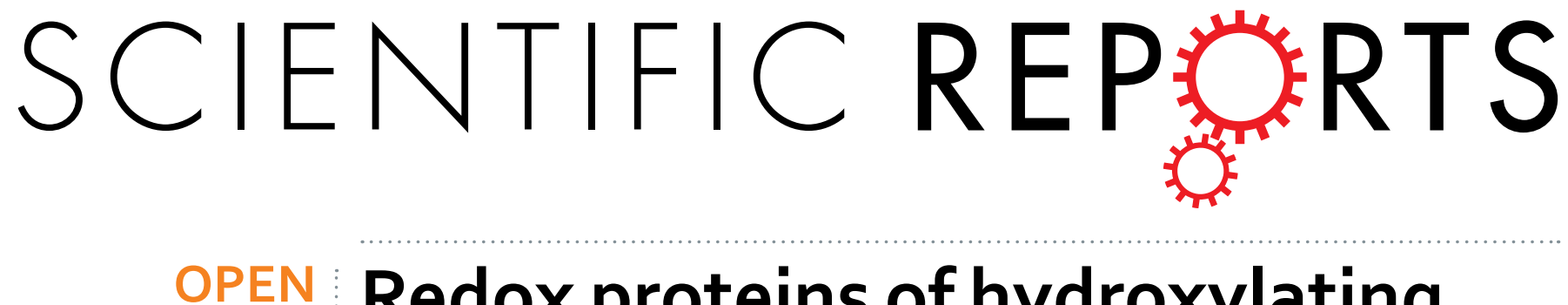

\title{
Redox proteins of hydroxylating bacterial dioxygenases establish a regulatory cascade that prevents
}

Received: 27 January 2016 Accepted: 09 March 2016 Published: 31 March 2016

\section{gratuitous induction of tetralin biodegradation genes}

Laura Ledesma-García ${ }^{1}$, Ana Sánchez-Azqueta ${ }^{2}$, Milagros Medina ${ }^{2}$, Francisca Reyes-Ramírez ${ }^{1}$ \& Eduardo Santero ${ }^{1}$

Bacterial dioxygenase systems are multicomponent enzymes that catalyze the initial degradation of many environmentally hazardous compounds. In Sphingopyxis granuli strain TFA tetralin dioxygenase hydroxylates tetralin, an organic contaminant. It consists of a ferredoxin reductase (ThnA4), a ferredoxin (ThnA3) and a oxygenase (ThnA1/ThnA2), forming a NAD(P)H-ThnA4-ThnA3-ThnA1/ThnA2 electron transport chain. ThnA 3 has also a regulatory function since it prevents expression of tetralin degradation genes $(t h n)$ in the presence of non-metabolizable substrates of the catabolic pathway. This role is of physiological relevance since avoids gratuitous and wasteful production of catabolic enzymes. Our hypothesis for thn regulation implies that ThnA3 exerts its action by diverting electrons towards the regulator ThnY, an iron-sulfur flavoprotein that together with the transcriptional activator ThnR is necessary for thn gene expression. Here we analyze electron transfer among ThnA4, ThnA3 and ThnY by using stopped-flow spectrophotometry and determination of midpoint reduction potentials. Our results indicate that when accumulated in its reduced form ThnA 3 is able to fully reduce ThnY. In addition, we have reproduced in vitro the regulatory circuit in the proposed physiological direction, $N A D(P) H-$ ThnA4-ThnA3-ThnY. ThnA3 represents an unprecedented way of communication between a catabolic pathway and its regulatory system to prevent gratuitous induction.

A wide range of aromatic compounds are major environmental pollutants, continuously discharged into the environment through industrial and urban activities causing irreversible damage to the biosphere. Microbial catabolic potential allows using a variety of hazardous compounds as growth substrates. Aromatic catabolic pathways usually initiate biodegradation by incorporation of oxygen into the aromatics rings catalyzed by Rieske non-haem iron diooxygenases, a reaction requiring oxygen and reducing equivalents from $\mathrm{NAD}(\mathrm{P}) \mathrm{H}$. These multicomponent enzymes consist of a reductase, an oxygenase and, in some cases, an additional ferredoxin that mediates electron transfer between the former two components ${ }^{1}$. Further metabolism is achieved through hydroxylated aromatic intermediates. An efficient catabolic process imposes its catabolic genes being expressed at adequate levels only when the right substrates, those that the catabolic pathway can metabolize, are present, thus avoiding energetically wasteful production of catabolic enzymes and potentially wasteful consumption of NAD $(P)$ $\mathrm{H}$. Regulators of catabolic pathways are quite promiscuous, responding to distinct sets of structural analogues substrates and even to non-aromatic pollutants in some aromatic pathways ${ }^{2}$. This may result in a detrimental gratuitous induction of the pathway.

Sphingopyxis granuli strain TFA is able to grow with tetralin as a sole source of carbon and energy. Tetralin $(1,2,3,4$-tetrahydronaphthalene) is a bicyclic molecule composed of an aromatic and an alicyclic moiety, which is

${ }^{1}$ Centro Andaluz de Biología del Desarrollo, Universidad Pablo de Olavide/Consejo Superior de Investigaciones Científicas/Junta de Andalucía, and Departamento de Biología Molecular e Ingeniería Bioquímica, Seville, Spain. ${ }^{2}$ Departamento de Bioquímica y Biología Molecular y Celular, and Instituto de Biocomputación y Física de Sistemas Complejos (BIFI), Universidad de Zaragoza, Zaragoza, Spain. Correspondence and requests for materials should be addressed to M.M. (email: mmedina@unizar.es) or F.R.R. (email: freyram@upo.es) 
found at low concentrations in different crude oils, and it is also industrially produced for its use as an organic solvent. The degradation pathway has been characterized both at the biochemical and genetic levels ${ }^{3-7}$. As observed for other aromatic compounds, degradation of tetralin is initiated by dioxygenation of the aromatic ring, which is catalyzed by the tetralin dioxygenase enzymatic complex. This complex consist of a NAD(P)H-dependent ferredoxin reductase ThnA4 (NCBI protein accession number AAN26446), a ferredoxin ThnA3 (AAD52963), and a Rieske-type dioxygenase ThnA1/ThnA2 (AAN26443; AAN26444) that hydroxylates tetralin, forming the NAD(P)H-ThnA4-ThnA3-ThnA1/ThnA2 electron transport chain.

Expression of the tetralin biodegradation genes $(t h n)$ in $S$. granuli requires expression of the thnR and thnY regulatory genes. ThnR (AAU12855) is a LysR-type transcriptional activator that activates thn gene transcription in response to tetralin by binding to sites present at each of the four thn promoter regions ${ }^{7,8}$. ThnY (AAU12856) contains FAD and a plant-type [2Fe-2S] cluster and shows spectral features of the bacterial oxygenase-coupled $\mathrm{NAD}(\mathrm{P}) \mathrm{H}$-dependent ferredoxin reductases. However, unlike ferredoxin reductases, purified ThnY is not reduced by $\mathrm{NAD}(\mathrm{P}) \mathrm{H}$ and instead has been recruited by the regulatory module. Addition of Thn $\mathrm{Y}_{\mathrm{ox}}$ to electrophoretic mobility shift assays containing ThnR and a probe bearing the thn promoters indicated that ThnY directly promotes thn transcription activation by $\mathrm{ThnR}^{9}$. In addition, the ferredoxin ThnA 3 adds an "extra" regulation by preventing expression of the thn genes when the inducer of the pathway is a poor substrate for the dioxygenase, avoiding gratuitous induction of the pathway. Thus, mutant strains lacking ThnA3 activate transcription of the catabolic pathway to high levels in response to compounds other than tetralin. On the other hand mutants lacking either the $\alpha$ (ThnA1) or $\beta$ (ThnA2) subunits of the dioxygenase showed very low levels of thn expression even in the presence of tetralin. Based on these data, it has been proposed that ThnA3 exerts its regulatory function based on a redox sensory mechanism. Thus, under conditions in which the catabolic pathway cannot efficiently metabolize the inducer molecule (deficient electron flux to the dioxygenase), ThnA3 accumulates in its reduced form and prevents induction of tetralin gene expression ${ }^{10,11}$. We have found that some ThnY variants with amino acid substitutions in the cofactor binding site completely loose the discrimination capacity of the thn system ${ }^{11}$. These results suggest a unique regulatory model whereby ThnA3 signals are transmitted to the regulatory system via modification of the redox state of ThnY, forming a new regulatory NAD $(\mathrm{P}) \mathrm{H}-\mathrm{Thn} \mathrm{A} 4-\mathrm{ThnA} 3-\mathrm{ThnY}$ electron transport chain.

By using stopped-flow spectrophotometry methods and determining midpoint reduction potentials of the implicated proteins, we demonstrate in this paper that the ferredoxin ThnA3 is the electron partner of ThnY. We have further analyzed the electron transfer processes between the NADP(H)-ThnA $4_{\text {ox }}$, ThnA $4_{\text {red }}-\operatorname{ThnA} 3_{\text {ox }}$ and $\mathrm{ThnA} 3_{\text {red }}-\mathrm{ThnY} \mathrm{ox}_{\mathrm{x}}$ couples, and reconstituted the electron transfer chain in the physiological direction proposed, ThnA4-ThnA3-ThnY. To our knowledge, this is the only case known where catabolic enzymes commonly associated with the electron transport chain of dioxygenase systems are coupled to the regulatory proteins to adjust gene expression in response to the catabolic flux in the cell.

\section{Results}

Electron transfer from ferredoxin ThnA $3_{\text {red }}$ to ThnY $Y_{\text {ox }}$. Previous in vivo studies using mutated ThnY lead to the proposal of a model in which the interaction of ThnA $3_{\text {red }}$ with ThnY negatively modulates ThnY activity, through the reduction of ThnY and its subsequent inactivation ${ }^{11}$. The in vitro demonstration of the functionality of the ThnA3-ThnY electron transfer process would strongly support the model for modulation of the regulatory system.

To investigate these facts, the hexahistidine-tagged versions of each of these proteins were purified by using metal chelate affinity and size exclusion chromatography. The $\mathrm{His}_{6}$-ThnY holoenzyme, containing FAD and a plant-type $[2 \mathrm{Fe}-2 \mathrm{~S}]$ cluster, was purified as previously reported ${ }^{9}$. ThnA3-His ${ }_{6}$ was here purified for the first time as a protein with an apparent molecular mass of $14 \mathrm{kDa}$, calculated from its mobility in SDS-PAGE, which agrees with the calculated from its coding sequence. The ThnA3 sequence bears the highly conserved metal-binding motif, $\mathrm{CXHX}_{15-17} \mathrm{CX}_{2} \mathrm{H}$, containing the two cysteines and two histidines that co-ordinate the Rieske-type [2Fe-2S] cluster. Solutions of purified $\mathrm{ThnA}_{\mathrm{ox}}$ were brown-coloured and have the typical absorption spectrum of a Rieske-type [2Fe-2S] cluster with maxima at 280,320, and $461 \mathrm{~nm}$, and a shoulder at $580 \mathrm{~nm}$, and became fully reduced by dithionite (Supplementary Fig. S1a). Hence, ThnA3 displays similar spectral properties to those of the related ferredoxins from benzene, toluene, biphenyl, carbazole and napthalene dioxygenase systems ${ }^{12}$. Nevertheless, cluster incorporation in recombinant ThnA3 was incomplete, with an average of $35 \%$ of [ $2 \mathrm{Fe}-2 \mathrm{~S}$ ] incorporation, an observation commonly associated with over-expressed iron-sulfur proteins ${ }^{13}$. Herein this percentage was considered to calculate the amount of holo-ThnA3 in our kinetic and potentiometric assays. The electron transfer process from $\mathrm{ThnA} 3_{\text {red }}$ to $\mathrm{ThnY} \mathrm{Y}_{\mathrm{ox}}$ was analyzed by using stopped-flow spectroscopy, a method that can provide information on specific steps in the electron transfer reaction sequence and, therefore, will only imply ThnA3 in its holoprotein form when observing electron transfer. To do that we mixed a $\sim 4$-fold excess of an in vitro photoreduced solution of ThnA3 with $\mathrm{ThnY}_{\mathrm{ox}}$, and evolution of the process was followed over the visible range observing a general decrease in the absorbance. As shown in Fig. 1a, the excess of ThnA $3_{\text {red }}$ was able to fully reduce $\mathrm{ThnY}_{\mathrm{ox}}$ under anaerobic conditions without detection of any traces of a semiquinone intermediate state. Different profiles for absorption evolution at $450 \mathrm{~nm}$ and $531 \mathrm{~nm}$ (inset Fig. 1a) as well as global analysis of the spectral evolution along the process were consistent with a two-step model, $\mathrm{A} \rightarrow \mathrm{B} \rightarrow \mathrm{C}$, where three spectroscopic species can be distinguished (Fig. 1b). The initial species, A, practically results in the spectrum of Thn $Y_{\mathrm{ox}}$. Conversion of $\mathrm{A}$ into $\mathrm{B}$ occurs with an observed rate constant, $k_{\mathrm{A} \rightarrow \mathrm{B}}$, of $17.6 \pm 1.5 \mathrm{~min}^{-1}$ under conditions of Fig. 1, with an absorbance decrease at the flavin band-I (around $450 \mathrm{~nm}$ ) consistent with the two-electron reduction of the FAD cofactor of ThnY. In agreement, the spectrum of species B is consistent with the [2Fe-2S] cluster of ThnY remaining in the oxidized state. Spectroscopic changes for the final transformation of species B into C agree with the subsequent reduction of this $[2 \mathrm{Fe}-2 \mathrm{~S}]$ cluster $\left(k_{\mathrm{B} \rightarrow \mathrm{C}}=4.6 \pm 0.6 \mathrm{~min}^{-1}\right)$. Thus, spectrum for species $\mathrm{C}$ is consistent with a fully reduced ThnY. 

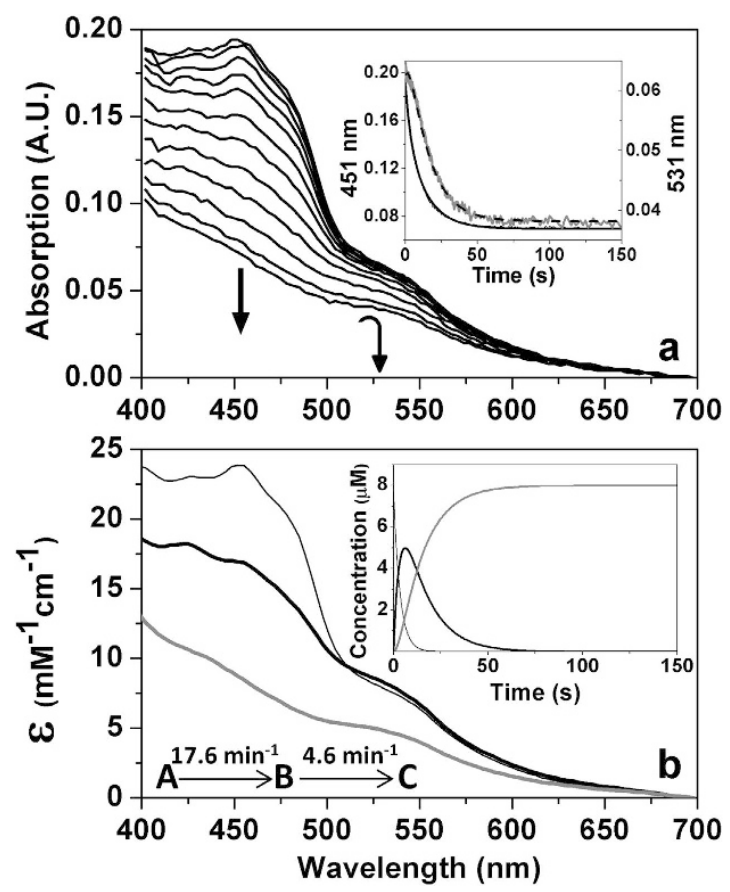

Figure 1. Anaerobic reduction of Thn $\mathrm{Y}_{\mathrm{ox}}$ by ThnA $\mathbf{3}_{\text {red }}$. (a) Spectral evolution of the reaction of Thn $\mathrm{Y}_{\mathrm{ox}}$ $(\sim 8 \mu \mathrm{M})$ with photoreduced $\mathrm{ThnA} 3_{\text {red }}(\sim 24 \mu \mathrm{M}$ holoenzyme) as measured by stopped-flow spectrophotometry. Spectra recorded at $0.00128,0.04736,0.4109,0.8614,1.778,3.488,5.382,8.25,11.74,17.8$, and $54.38 \mathrm{~s}$ after mixing are shown. Direction of the spectral evolution is indicated by arrows. The inset shows the absorbance evolution at $451 \mathrm{~nm}$ (black line) and $531 \mathrm{~nm}$ (grey line) and their corresponding global fits to a two-steps model, $\mathrm{A} \rightarrow \mathrm{B} \rightarrow \mathrm{C}$ (bold black lines). (b) Spectroscopic properties of the intermediate pre-steady-state species. The inset shows the evolution of the obtained spectral species over the time. Species A, B and C are shown as continuous black thin, black bold and grey bold lines, respectively. Measurements carried out in potassium phosphate $50 \mathrm{mM}, \mathrm{pH} 7.4, \mathrm{NaCl} 10 \mathrm{mM}$, glycerine $5 \%$.

These results evidence that ThnA 3 and ThnY are able to interact in solution and that ThnA $3_{\text {red }}$ is able to directly transfer electrons to the regulatory protein ThnY. Moreover, our results indicate that this electron transfer follows an ordered mechanism, with FAD reduction of ThnY occurring first and being its iron sulfur-center the one reduced in the final step.

Reduction of ferredoxin reductase ThnA4 ${ }_{\text {ox }}$ by $\mathrm{NAD}(\mathrm{P}) \mathrm{H}$. Ferredoxin reductases of the dioxygenases systems are known to supply reducing equivalents from $\mathrm{NAD}(\mathrm{P}) \mathrm{H}$ either directly to the terminal dioxygenase (Class I, two-component dioxygenase systems) or via an intermediary ferredoxin (Class II and III, three-component systems) ${ }^{1}$. In vivo, in the tetralin dioxygenase enzyme complex, (ThnA4-ThnA3-ThnA1/ ThnA2), the ferredoxin reductase ThnA4 is postulated to transfer reducing equivalents from $\mathrm{NAD}(\mathrm{P}) \mathrm{H}$ to ferredoxin ThnA $3^{6}$. ThnA 4 encodes a 339 -amino acid polypeptide, $45 \%$ identical to the ferredoxin reductases of the class III dioxygenase systems. These reductases share an $\mathrm{N}$-terminal domain that contains a conserved Cys- $X_{4}$-Cys- $X_{2}$-Cys- $X_{29 / 30}$-Cys motif that binds a plant-type [2Fe-2S] cluster, while the central and C-terminal domains contain the conserved motifs for flavin and NAD $(\mathrm{P}) \mathrm{H}$ binding, respectively ${ }^{9}$. To in vitro analyze the reduction of ThnA3 under conditions closer to the physiological ones, (NAD(P)H-ThnA4-ThnA3), we also produced for first time recombinant ThnA4- $\mathrm{His}_{6}$ purified at homogeneity and studied its ability to accept electrons from pyridine nucleotides. Although ThnA4-His ${ }_{6}$ loses significant amounts of its flavin FAD cofactor during purification, upon FAD reconstitution its UV-visible air-oxidized spectrum revealed the typical absorption maxima at 276, 370, 421, and $461 \mathrm{~nm}$, and a shoulder at about $550 \mathrm{~nm}$, the later disappearing upon reduction by dithionite (Supplementary Fig. S1b). Thus, reconstituted ThnA4- $\mathrm{His}_{6}$ contains the expected FAD and [2Fe-2S] redox centers, showing similar spectral features to the reductases from the benzoate 1,2 -dioxygenase ${ }^{14}$, napthalene dioxygenase ${ }^{15}, 2$-halobenzoate 1,2-dioxygenase ${ }^{16}$, and carbazole dioxygenase $\mathrm{e}^{17}$ systems.

Reduction of ThnA 4 ox by NAD(P)H was also analyzed by fast-kinetic stopped-flow. Spectral evolution after mixing ThnA $4_{\text {ox }}$ with NADH under anaerobic conditions clearly showed reduction of the enzyme (Fig. 2a). Similar results were also obtained under aerobic conditions and when NADPH was used as electron donor (not shown), indicating that ThnA $4_{\text {ox }}$ can catalyze the oxidation of both coenzymes. Decrease in the absorbance at $461 \mathrm{~nm}$ upon reaction was concomitant with the appearance of a broad small long-wavelength band centered at $625 \mathrm{~nm}$ consistent with the appearance of a flavin-nicotinamide charge transfer complex along the reaction that finally bleaches (Fig. 2a,b). Global analysis of the spectral evolution shown in Fig. 2a was consistent with a two-step model (Fig. 2b,c). The first step, A $\rightarrow$ B, accounted for bleaching of the flavin band with the concomitant appearance of the long-wavelength charge transfer band. Transformation of B into C occurred with a rate 

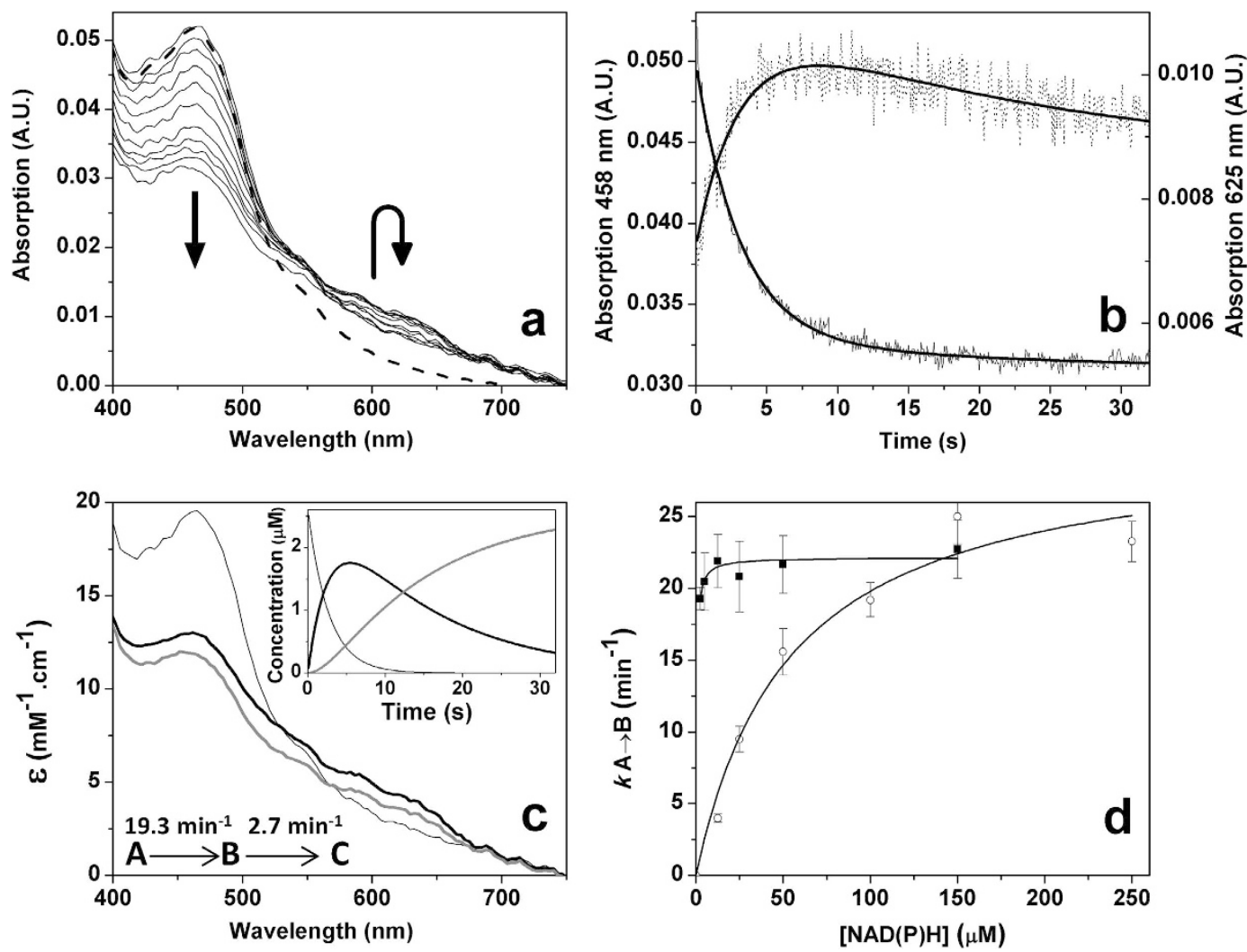

Figure 2. Reduction of ThnA4 $4_{\text {ox }}$ by NADH. (a) Spectral evolution of ThnA4 $(\sim 2.5 \mu \mathrm{M})$ along reaction with $\mathrm{NADH}(\sim 2.5 \mu \mathrm{M})$ as measured by stopped-flow spectroscopy under anaerobic conditions. The thick dashed black line corresponds to the spectrum of ThnA $4_{\text {ox }}$ before mixing. Spectra recorded at $0.08064,0.1626,0.3264$, $0.8179,1.309,2.456,3.931,5.242,7.29,10.07$, and $30.23 \mathrm{~s}$ after mixing are shown. Directions of the spectral evolutions are indicated by arrows. (b) Evolution of the absorbance at $458 \mathrm{~nm}$ (line) and $625 \mathrm{~nm}$ (dotted line) and their corresponding global fits (bold lines) to a two-steps model, $\mathrm{A} \rightarrow \mathrm{B} \rightarrow \mathrm{C}$. (c) Spectroscopic properties of the intermediate pre-steady-state species. The inset shows the evolution of the obtained spectral species over the time. Species A, B and C are shown as continuous black thin, black bold and grey bold lines, respectively. (d) Observed $k_{\mathrm{A} \rightarrow \mathrm{B}}$ values as a function of NADH (filled squares) and NADPH (open circles) concentrations. Lines represent data fit to Eq. 1. Experimental conditions as in Fig. 1.

constant $\sim 7$-fold slower than the initial process and accounted for the disappearance of the charge transfer complex band (Fig. 2a-c). When we used large coenzyme concentrations, and after a relatively long lag phase, conversion of $\mathrm{C}$ into a final $\mathrm{D}$ species was also observed, with spectral changes consistent with achieving full reduction of FAD and [2Fe-2S] clusters (not shown).

$k_{\mathrm{A} \rightarrow \mathrm{B}}$ values showed a saturation profile on $\mathrm{NADH}$ concentration that allowed us to estimate a limiting hydride transfer rate constant from NADH to ThnA4 $4_{\text {ox }}, k_{\text {red }}{ }^{\mathrm{NADH}}$, of $22.1 \pm 2.3 \mathrm{~min}^{-1}$, while suggesting a $K_{\mathrm{d}}{ }^{\mathrm{NDH}}$ value lower than $0.4 \mu \mathrm{M} . k_{\mathrm{A} \rightarrow \mathrm{B}}$ values for NADPH showed a concentration saturation dependence that allowed fitting of the data to the equation describing binding at a single site followed by the hydride transfer processes and determination of the NADPH:ThnA4 $4_{\text {ox }}$ dissociation constant $\left(K_{\mathrm{d}}^{\mathrm{NADPH}}, 54 \pm 13 \mu \mathrm{M}\right)$ and the hydride transfer rate from NADPH to the FAD cofactor $\left(k_{\text {red }}{ }^{\mathrm{NADPH}}=30.5 \pm 2.1 \mathrm{~min}^{-1}\right)$ (Fig. 2d). These parameters indicate that despite limiting rate constants for hydride transfer to ThnA $4_{\text {ox }}$ are very similar for both coenzymes, the affinity of Thn $4_{\text {ox }}$ for NADH is considerably higher than that for NADPH. These data are consistent with a higher efficiency for the process with $\mathrm{NADH}$, indicating it as the preferred physiological hydride donor to ThnA $4_{\mathrm{ox}}$. Similar results were obtained regardless using aerobic or anaerobic conditions and the reverse reaction was undetectable under our experimental conditions, with the only exception of a very slow reverse reaction $\left(k_{\text {reox }}=0.16 \mathrm{~min}^{-1}\right)$ for the process with NADPH under aerobic conditions. These results agree with observations in other related systems where the reductases, such as the ones of phthalate, toluene and benzene dioxygenases, are very specific for $\mathrm{NADH}^{18-20}$, while others less specific showed also preference for $\mathrm{NADH}$ over $\mathrm{NADPH}^{15}$.

Reduction of ferredoxin ThnA3 $3_{\text {ox }}$ by ferredoxin reductase ThnA4. Once demonstrated that ThnA4 is functionally reduced by $\mathrm{NAD}(\mathrm{P}) \mathrm{H}$, we also analyzed its ability to transfer electrons from $\mathrm{NADH}$ to $\mathrm{ThnA} 3_{\text {ox }}$. With this aim we followed the spectral evolution upon mixing under anaerobic conditions an excess of ThnA3 ${ }_{\text {ox }}$ with ThnA $4_{\text {red }}$, which was formed by previous incubation of ThnA $4_{\text {ox }}$ with NADH (Fig. 3). The spectral shape of ThnA $3_{\text {ox }}$ rapidly changed after mixing with ThnA $4_{\text {red }}$ and its absorption peaks were displaced to 435 and $522 \mathrm{~nm}$ (Fig. 3a). These absorbance maxima are characteristic of reduced Rieske-type ferredoxins of aromatic 

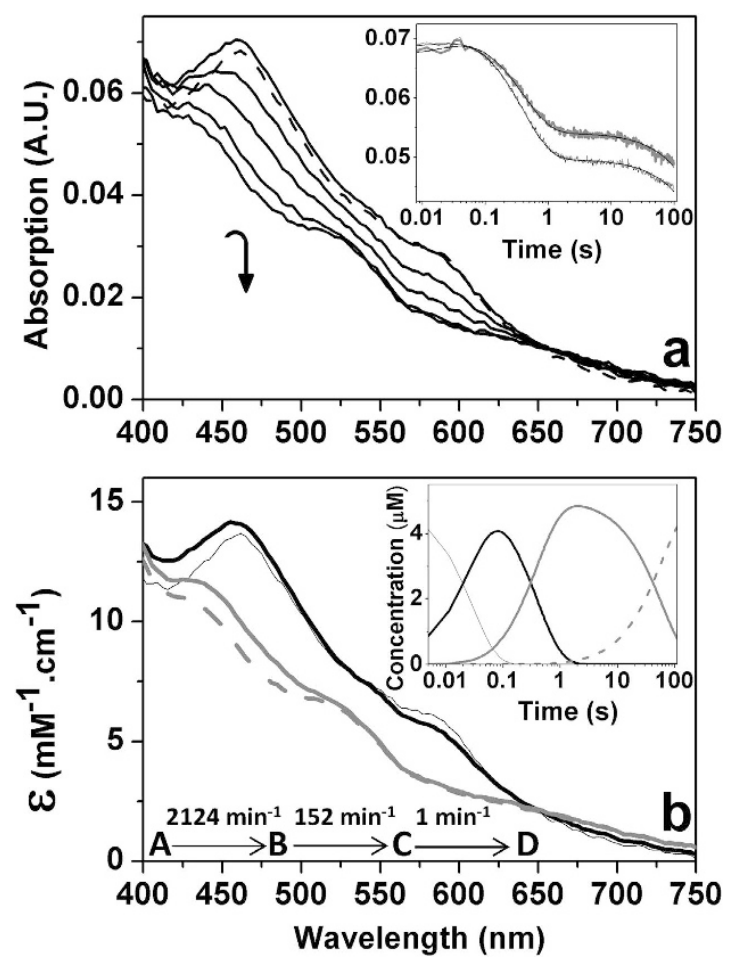

Figure 3. Anaerobic reduction of ThnA3 $3_{\mathrm{ox}}$ by ThnA4 $4_{\text {red }}$. (a) Spectral evolution of the reaction of ThnA $3_{\mathrm{ox}}$ $\left(\sim 14 \mu \mathrm{M}\right.$ holoenzyme) with ThnA $4_{\text {red }}(\sim 5 \mu \mathrm{M}$, previously reduced with $50 \mu \mathrm{M}$ NADH) as measured by stoppedflow spectroscopy under anaerobic conditions. Spectra recorded at 0.00384 (dashed line), 0.03968, 0.2138, $0.5082,22.14$, and $220.8 \mathrm{~s}$ after mixing are shown. The inset shows the evolution of the absorbance at $452 \mathrm{~nm}$ (grey bold line) and $462 \mathrm{~nm}$ (thin black line), as well as their corresponding global fits (bold lines) to a threesteps model, $\mathrm{A} \rightarrow \mathrm{B} \rightarrow \mathrm{C} \rightarrow \mathrm{D}$. (b) Spectroscopic properties of the intermediate pre-steady-state species. The inset shows the evolution of the obtained spectral species over the time. Species A, B, C and D are shown as continuous black thin, black bold, grey bold and grey dashed lines, respectively. Experimental conditions as in Fig. 1.

systems such as BphA3 of the biphenyl dioxygenase complex from Pseudomonas sp. KKS102 ${ }^{21}$, the ferredoxin NAP component of naphthalene dioxygenase from Pseudomonas ${ }^{17}$ and the CarAc component of the carbazole $1,9 \alpha$-dioxygenase ${ }^{15}$. The overall process fit to a three-step model (Fig. 3a, inset, and 3b). Species A resembled the ThnA $3_{\text {ox }}$ spectrum. Transition of A into B was related with a slight increment in the absorption of the 460 band and a decrease in the $600 \mathrm{~nm}$ band, changes consistent with the production of the interaction between both proteins. Transition of $\mathrm{B}$ into $\mathrm{C}$ related with absorption decrease in the whole wavelength range consistent with reduction of ThnA $3_{\text {ox }}$ by the NADH reduced ThnA4. Transformation of species C into D is a considerably slower process that accounts for a very small change in amplitude, probably related with final consumption of the excess of reduced coenzyme and the achievement of the steady-state equilibrium.

Altogether these results indicate that ThnA4 functions as a conventional $\mathrm{NAD}(\mathrm{P}) \mathrm{H}$-dependent oxidoreductase of the dioxygenase systems, accepting a hydride from $\mathrm{NAD}(\mathrm{P}) \mathrm{H}$ by its flavin cofactor and transferring the two obtained electrons one at a time through its [2Fe-2S] cluster to the one-electron acceptor ThnA3 ferredoxin.

Reconstitution of the regulatory electron transfer NADH-ThnA4-ThnA3-ThnY chain. Since $\mathrm{ThnA} 3_{\text {red }}$ is able to reduce $\mathrm{ThnY}_{\text {ox }}$ when its iron cluster is photoreduced (Fig. 1) and NADH reduced ThnA4 (Fig. 2) is able to reduce ThnA $3_{\text {ox }}$ (Fig. 3), we finally attempted to in vitro reconstitute the regulatory electron-transport chain in the proposed physiological direction by testing the reduction of the regulatory Thn $\mathrm{Y}_{\text {ox }}$ by NADH via ThnA4 and ThnA3. Results for the anaerobic reconstitution of this system are shown in Fig. 4. Anaerobic stopped-flow measurements were carried out after mixing ThnA $4_{\text {red, }}$ reduced by preincubation with $\mathrm{NADH}$, with a mixture containing $\mathrm{ThnA} 3_{\mathrm{ox}}$ and $\mathrm{ThnY} \mathrm{Y}_{\mathrm{ox}}$. Spectral changes clearly indicated that Thn $\mathrm{Y}_{\mathrm{ox}}$ became completely reduced along the reaction time-course. Global analysis of the spectral evolution fit to a four-step model, $\mathrm{A} \rightarrow \mathrm{B} \rightarrow \mathrm{C} \rightarrow \mathrm{D} \rightarrow \mathrm{E}$ (Fig. 4a, inset and $\mathrm{b}$ ). The initial intermediate species, $\mathrm{A}$, is consistent with a mixture that mainly contains $\mathrm{ThnA} 3_{\mathrm{ox}}, \mathrm{ThnY}_{\mathrm{ox}}$ and ThnA $4_{\text {red }}$. Conversion of A into B shows a slight absorbance increase that can be related with complex formation among the proteins present in solution. Conversion of $\mathrm{B}$ into $\mathrm{C}$ is consistent with reduction of the $[2 \mathrm{Fe}-2 \mathrm{~S}]$ center of ThnA3 with a conversion rate constant, $k_{\mathrm{B} \rightarrow \mathrm{C}}=110 \mathrm{~min}^{-1}$, very similar to that above obtained for the reduction of ThnA $3_{\text {ox }}$ by ThnA $4_{\text {red }}$ in the absence of ThnY (Fig. 3). Conversion of $\mathrm{C}$ into $\mathrm{D}$ indicates reduction of the $\mathrm{FAD}$ of ThnY, with an observed rate constant, $k_{\mathrm{C} \rightarrow \mathrm{D}}=15 \mathrm{~min}^{-1}$, in the range described above by the first step in the analysis of the reduction of Thn $\mathrm{Y}_{\mathrm{ox}}$ by photoreduced ThnA3 (Fig. 1). Evolution to species E indicates final reduction of the three proteins with an observed rate constant of 

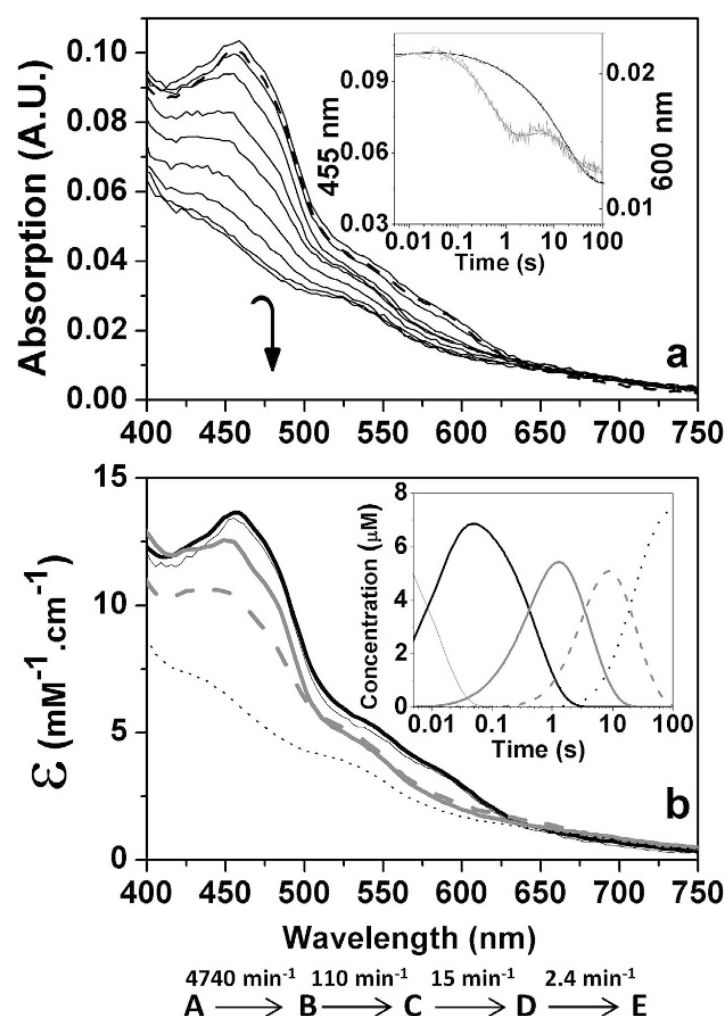

Figure 4. Reconstitution of the complete electron transfer chain: anaerobic reduction of Thn $\mathrm{Y}_{\mathrm{ox}}$ by NADH throughout ThnA $3_{\text {ox }}$ and ThnA4 ${ }_{\text {ox }}$ (a) Spectral evolution of the mixing ThnA $4_{\text {red }}(\sim 2.5 \mu \mathrm{M}$ of protein preincubated with $50 \mu \mathrm{M}$ NADH, in syringe 1$)$ with a mixture containing ThnA $3_{\text {ox }}(\sim 25 \mu \mathrm{M}$ holoenzyme) and $\mathrm{ThnY}_{\mathrm{ox}}(\sim 7.5 \mu \mathrm{M})$ (in syringe 2$)$ in the stopped-flow equipment under anaerobic conditions. Spectra at 0.00384 , $0.03968,0.754,0.887,4.317,8.982,18.68,38.31,103$, and $197 \mathrm{~s}$ after mixing are shown. The inset shows the spectral evolution at $455 \mathrm{~nm}$ (black line) and $600 \mathrm{~nm}$ (grey line), as well as and the corresponding global fits (bold lines) to a four-steps model, $\mathrm{A} \rightarrow \mathrm{B} \rightarrow \mathrm{C} \rightarrow \mathrm{D} \rightarrow \mathrm{E}$. (b) Spectroscopic properties of the intermediate presteady-state species. The inset shows the evolution of the obtained spectral species over the time. Species A, B, $\mathrm{C}, \mathrm{D}$ and $\mathrm{E}$ are shown as continuous black thin, black bold, grey bold, grey dashed and dotted lines, respectively. Experimental conditions as in Fig. 1.

$2.4 \mathrm{~min}^{-1}$, again comparable with the values for the second steps of both the reduction of Thn $\mathrm{Y}_{\text {ox }}$ by ThnA $3_{\text {red }}$ (Fig. 1) and of ThnA $3_{\text {ox }}$ by ThnA $4_{\text {red }}$ (Fig. 3). Similar results were obtained under aerobic conditions, although in the presence of air the spectrum of Thn $\mathrm{Y}_{\mathrm{ox}}$ resulted regenerated at the end of the reaction. Control experiments in which $\mathrm{ThnY}_{\mathrm{ox}}$ reduction was monitored in the absence of ThnA3, both under the same experimental conditions as well as in the presence of oxygen, showed that ThnA $4_{\text {red }}$ was unable to reduce ThnY $\mathrm{Y}_{\text {ox }}$. These results confirm i) the requirement of ThnA3 for the efficient reduction of $\mathrm{ThnY}_{\mathrm{ox}}$, as well as ii) the fact that we have reproduced in vitro the regulatory electron transfer chain from $\mathrm{NADH}$ to $\mathrm{ThnY}_{\mathrm{ox}}$ via ThnA4 and ThnA3, as it is proposed to occur in vivo.

Redox potentials values of ThnA4, ThnA3 and ThnY. Midpoint reduction potentials $\left(E_{\mathrm{m}}\right)$, for ThnA3, $\mathrm{ThnY}$ and ThnA4, were determined by potentiometric titration and analysis of their visible spectra upon anaerobic photoreduction (Fig. 5). Spectral characteristics along photoreduction of ThnA3 were consistent with reduction of the $[2 \mathrm{Fe}-2 \mathrm{~S}]$ cluster in a process exchanging a single electron (Fig. 5a). Global fitting of data at several wavelengths to Eq. 2 allowed determination of a midpoint potential, $E_{\text {ThnA3SFeox/red }}$ of $-112 \pm 5 \mathrm{mV}$ (Fig. $5 \mathrm{a}$ inset). Spectra recorded upon photoreduction of ThnY showed the almost simultaneous reduction of its FAD (flavin band-I decrease at $454 \mathrm{~nm}$ ) and [2Fe-2S] (absorption decrease at $530 \mathrm{~nm}$ ) redox cofactors (Fig. 5b). Additionally, an absorption evolution at $380 \mathrm{~nm}$ consistent with the appearance of traces of anionic semiquinone along reduction was observed. Nevertheless, we observed that its maximal stabilization was too small to expect independent determination of the midpoint reduction potentials for the two individual oxido-reduction couples $\left(E_{\mathrm{ox} / \mathrm{sq}}\right.$ and $\left.E_{\mathrm{sq} / \mathrm{hq}}\right)$. The plot of different wavelengths relative absorptions versus the measured potentials was globally fit to Eq. 3, allowing determination of $E_{\text {ThnYFADox/hq }}$ and $E_{\text {ThnYSFeox/red }}$ with values of $-131 \pm 8 \mathrm{mV}$ and $-136 \pm 8 \mathrm{mV}$, respectively (Fig. 5b inset). Photoreduction of ThnA4 resulted in the extremely slow stabilization of the potential values after each reduction step, increasing the experimental time and the protein denaturation along the experiment. This enforced us to measure potentials and their corresponding absorption spectra at fixed times after each illumination event, that prevented accurate determination of midpoint potentials. Therefore, this experiment only suggested that $E_{\mathrm{ThnA} 4 \mathrm{FAD} \text { ax/hq }}$ and $E_{\mathrm{ThnA} 4 \mathrm{SF} F o x / \text { red }}$ values must be in the $-200 \mathrm{mV}$ to $-150 \mathrm{mV}$ ranges respectively. 


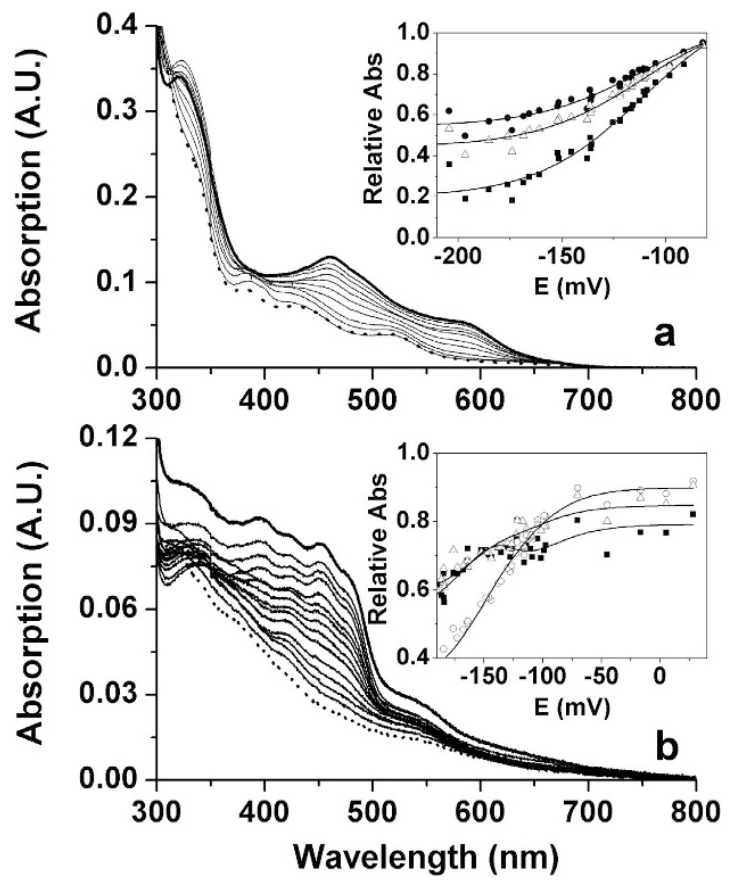

Figure 5. Potentiometric titrations of ThnA3 and ThnY. Spectral changes during photoreduction of (a) ThnA3 $(\sim 20 \mu \mathrm{M})$ and $(\mathbf{b})$ ThnY $(\sim 5 \mu \mathrm{M})$. Buffers were supplied with 5-deazariboflavin, EDTA and the corresponding chemical mediators. Titrations were carried out at $15^{\circ} \mathrm{C}$ in potassium phosphate $50 \mathrm{mM}, \mathrm{pH}$ 7.4 for ThnA3 and in 0.1 M HEPES, pH 7.4 for ThnY. Arrows indicate direction of spectral changes. The insets show multiple wavelength variation of the relative absorptions plotted against the redox potential of the solution $(\mathrm{mV} / \mathrm{SHE})$ at; (a) $590(\mathbf{\bullet}), 520(\bullet)$ and $462(\Delta) \mathrm{nm}$ for ThnA3 and (b) $530(\mathbf{\bullet}), 450(\mathrm{\circ})$ and $380(\Delta) \mathrm{nm}$ for ThnY. Continuous lines show simultaneous fits of the different wavelength data to Eq. 2 for ThnA3 and Eq. 3 for ThnY.

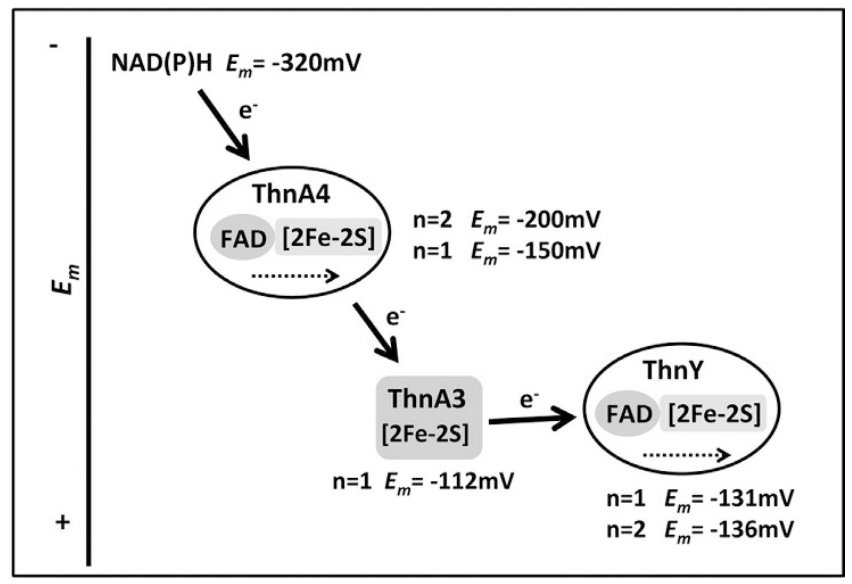

Figure 6. In vivo electron transfer pathway proposed for the reduction of ThnY by NAD(P)H via ThnA4ThnA3. Midpoint reduction potentials are indicated for each redox cofactor. Complete results for their determination are shown in Fig. 5. Inter and intramolecular electron transfers are represented by arrows.

A schematic diagram with the midpoint reduction potentials and the inter and intra-molecular electron transfer steps is shown in Fig. 6. The ThnY midpoint reduction potential is slightly more electronegative than that of ThnA3, thus indicating that electron transfer in the direction ThnA3 $\rightarrow$ ThnY is only possible when ThnA3 accumulates in its reduced form (such condition will displace the actual reduction potential of ThnA3 to more negative values than the one determined as midpoint potential).

\section{Discussion}

A feature of the regulatory systems of many biodegradation pathways is that the range of inducer molecules to which they respond is not the same as the range of substrates that the catabolic pathway can transform, 


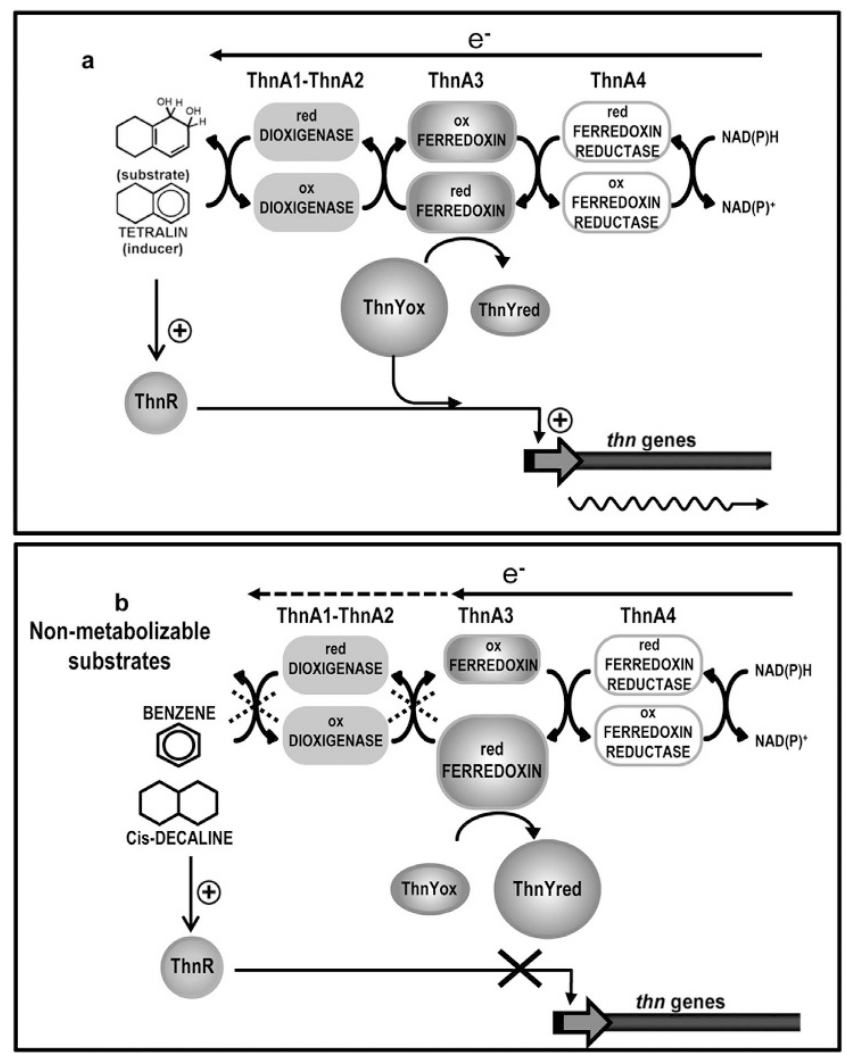

Figure 7. Model for the regulation of thn genes in response (a) to tetralin and (b) to non-metabolizable substrates. Blockage of electron transfer is represented by dotted crosses. The sizes of the circles indicate the relative abundance of that form of the protein according to the substrates supplied.

therefore resulting in a superfluous and energetically wasteful production of catabolic enzymes unable to use the non-metabolizable molecules. Some regulators recognize as effectors molecules those with structural analogy to the substrate or even quite dissimilar compounds. Representative examples are DmpR and XylR, $\sigma^{54}$-dependent regulators for catabolism of aromatics hydrocarbons such as (methyl)phenol and toluene/xylene respectively, which exhibit a very broad effector specificity ${ }^{2}$. Other catabolic pathways in order to prevent uncoordinated induction express the biodegradation genes not in response to the substrate but to some intermediate in the catabolism of the substrate $e^{2,22,23}$. However, this response implies high basal levels of expression to accumulate sufficient inducer intermediate to allow substantial degradation of the substrate. In addition, gratuitous induction is not fully prevented since some inducer intermediates may be produced through different peripheral routes that use different catabolic substrates.

The in vivo model for thn gene regulation presented in Fig. 7 proposes that ThnA3 reports to the regulatory ThnR-ThnY system whether a potential inducer molecule is also a good substrate of the catabolic pathway, based in a redox sensory mechanism ${ }^{10}$. In recent years, much progress has been made in understanding how Fe-S clusters regulatory proteins reprogram the expression of genes in response to environmental stimuli. A challenging question is to relate both the in vitro reactions of Fe-S clusters with its physiological relevance ${ }^{24}$. Our model is quite unique, since ThnA3 is the only ferredoxin that takes part in oxidative hydroxylation of aromatic compounds known to be involved in regulation of gene expression. Therefore, it is a crucial question to elucidate the mechanism by which ThnA3 exerts its function.

The expression phenotypes of the ThnY mutants have provided genetic evidences indicating that control of the ThnY redox state is essential for efficient regulation of thn genes, since some of the thn $Y$ mutations in the electron cofactor binding sites alter the range of molecules able to activate the catabolic pathway. In this way, thn $Y$ mutant strains behave as the mutants lacking ThnA3, expressing thn genes in the presence of not suitable molecules such as cis-decalin, cyclohexane, trans-decalin, or benzene ${ }^{11}$. These findings and the in vivo model imply that under certain circumstances, electrons from $\mathrm{NAD}(\mathrm{P}) \mathrm{H}$ that are accumulated in ThnA3 are redirected towards ThnY instead of the dioxygenase, (NAD(P)H-ThnA4-ThnA3-ThnY electron chain), thus resulting in ThnY inactivation. To provide biochemical evidences of this regulatory electron transport chain, we have characterized the sequence for electron transport in this system. Our results clearly show that when the [2Fe-2S] cluster of ThnA3 is photoreduced or reduced by its physiological $\operatorname{NADP}(\mathrm{H})$ electron donor (ThnA4), it is able to reduce both the flavin and the $[2 \mathrm{Fe}-2 \mathrm{~S}]$ cluster of ThnY, thus strongly supporting the proposed regulatory model for the regulation of thn.

According to this model, ThnA3 is predominantly in its oxidized form in the presence of tetralin (Fig. 7a), the real substrate of the catabolic pathway. Reduction of ThnY by ThnA3 is minimal under these conditions, since 
electrons would be preferentially transferred to the dioxygenase, thus allowing ThnR and ThnY $\mathrm{ox}_{\text {in }}$ to activate the thn promoters. In the absence of an efficient substrate that acts as an electron sink through the dioxygenation reaction (Fig. 7b), ThnA3 is accumulated in its reduced state. As a result, reduction of ThnY $\mathrm{O}_{\mathrm{ox}}$ by ThnA $3_{\text {red }}$ will take place, switching ThnY into an abundant reduced form, thus impairing thn gene transcription.

Analogous proteins to ThnA4 and ThnA3 have been reported to function in the multienzyme systems that dioxygenate the aromatic substrates to cis-dihydrodiols. ThnA4 has several properties in common with the three-component oxygenase systems that catalyze reduction of ferredoxin from $\mathrm{NAD}(\mathrm{P}) \mathrm{H}$ : similar molecular weight, two prosthetic groups in a single polypeptide, a loosely bound molecule of FAD, and preference toward $\mathrm{NADH}$. In fact the expected range for midpoint potentials for ThnA4 $\left(E_{\mathrm{ThnA} 4 \mathrm{FAD} \text { ox/hg }}\right.$ and $\left.E_{\mathrm{ThnA} 4 \mathrm{SFeox} / \mathrm{red}}\right)$ is in agreement with its role as $\mathrm{NAD}(\mathrm{P}) \mathrm{H}$ ferredoxin reductase. For comparison, in the phthalate dioxygenase reductase, $\mathrm{PDR}$, the $E_{m}$ for flavin is $-230 \mathrm{mV}$, and the one-electron potential of [ $\left.2 \mathrm{Fe}-2 \mathrm{~S}\right]$ is $-174^{18}$. Similarly ThnA3 shares common properties to the ferredoxin type protein of multicomponent dioxygenases enzymes, they are all one electron carriers, each has a Rieske-type [2Fe-2S], have similar molecular weights and they are rather specific for the dioxygenase system ${ }^{1}$. However, reduction potentials of the dioxygenase ferredoxins ${ }^{25}$ are approximately $-150 \mathrm{mV}$. The $E_{\mathrm{ThnA} 3 \mathrm{ox} / \mathrm{red}}(-112 \mathrm{mV})$ is also comparable although clearly less negative than those found in ferredoxin Rieske counterparts. For comparison, the $E_{\mathrm{m}}$ for Rieske-type ferredoxins of aromatics benzene and biphenyl dioxygenases, or toluene 4-monooxygenase are $-155,-157$, and $-173 \mathrm{mV}$, respectively ${ }^{26-28}$. On the other hand, the $E_{\text {ThnYox/red }}(-131$ and $-136 \mathrm{mV})$ are less negative too than those of other ferredoxin reductases counterparts and slightly more negative than that of ThnA3 (Fig. 6). These values indicate that although when looking at midpoint reduction potentials electron transfer in the direction ThnA3 to ThnY is not favored, the situation can change when ThnA3 accumulates in the reduced form and its effective reduction potential becomes more negative. Moreover, these midpoint potentials fit well with the notion that $\mathrm{Thn} \mathrm{Y}_{\mathrm{ox}}$ should not efficiently compete with the dioxygenase for the electrons coming from ThnA3 and, therefore, ThnY should only be reduced when ThnA $3_{\text {red }}$ is highly accumulated in vivo and the equilibrium for the redox reaction changes. Actually, non-favored electron transfer from ThnA3 to ThnY may probably be essential for an efficient gene regulation because otherwise ThnY could always be reduced by $\mathrm{ThnA} 3_{\text {red }}$, thus preventing transcription of thn genes under all conditions.

In conclusion, in vivo expression of the thn genes is prevented when reductive inactivation of ThnY occurs. This may happen (i) in the presence of an inducer molecule that is not a substrate of the dioxygenase and consequently high levels of Thn $A 3_{\text {red }}$ are accumulated ${ }^{10}$, (ii) in the thnA1 or thnA2 mutant strains, lacking of the dioxygenase subunits, in which all ThnA $3_{\text {red }}$ is fully available to interact with ThnY and thn expression is impaired even in the presence of tetralin ${ }^{10}$ and (iii) when the inducer is also a substrate but its concentration is very low. Thus, under these conditions, redox equilibria favor electron transfer in the direction NADH $\rightarrow$ ThnA $4_{\mathrm{FAD}} \rightarrow$ ThnA4 ${ }_{[2 \mathrm{Fe}-2 \mathrm{~S}]} \rightarrow \mathrm{ThnA}_{[2 \mathrm{Fe}-2 \mathrm{~S}]} \rightarrow \mathrm{ThnY}_{\mathrm{FAD}} \rightarrow \mathrm{ThnY}_{[2 \mathrm{Fe}-2 \mathrm{~S}]}$ that we have reproduced in vitro. This way, the redox regulation exerted through ThnA3 not only prevents gratuitous induction by a non-metabolizable molecule but also may finely adjust the level of transcription of thn genes to the availability of the substrate.

\section{Methods}

Plasmids and strains constructions. Plasmids, strains, and primers used in this work are listed in Supplementary Table S1.

Plasmid pMPO785 to overproduce $\mathrm{His}_{6}$-ThnY was performed by PCR amplification of thnY with primers NdeI-thnY2 (5'AAAAACATATGGAAATCACCCTCATCC $3^{\prime}$ ) and thnY-BamHI (5'AAAAAGGATCCTTACGAAACAGAAAAATGGTAAGG3') using pMPO750 as template. thn $Y$ was cloned into pET-14b, PCR product and vector were cleavage with $\mathrm{NdeI}+\mathrm{BamHI}$.

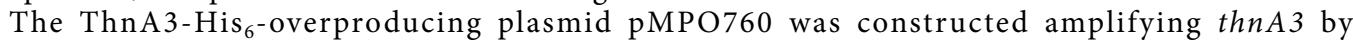
PCR with primers NdeI-thnA3 (5'CACATATGGGACGTAAGGTTAG3') and thnA3-XhoI (5'GGCTCGAGATCAAGATCCGCGA3'), using pMPO751 as the template. The PCR product was cleaved with $N d e I+X h o I$ and cloned into pET23a digested with the same enzymes.

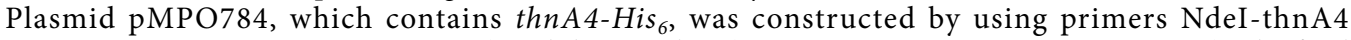
(5'GACATATGGGCAGCGCGCGCAT3') and thnA4-XhoI (5'GCCTCGAGGACAAAGCTGTC3'). The final DNA fragment was digested with NdeI + XhoI, and was introduced into pET23b digested with the same enzymes.

E. coli $\mathrm{DH} 5 \mathrm{\alpha}$ was used as a host in all cloning procedures ${ }^{40}$. All DNA manipulations were performed according to standard procedures ${ }^{29}$. Plasmid DNA was transferred to competent cells of $E$. coli strains by heat shock transformation. When required, antibiotics were used at the following concentrations: ampicillin, $100 \mu \mathrm{g} / \mathrm{ml}$, chloramphenicol, $15 \mu \mathrm{g} / \mathrm{ml}$, and gentamicin, $10 \mu \mathrm{g} / \mathrm{ml}$.

Purification of recombinant proteins. Expression and purification of $\mathrm{His}_{6}$-ThnY (containing an $\mathrm{N}$-terminal $\mathrm{His}_{6}$ tag), ThnA3-His ${ }_{6}\left(\mathrm{C}\right.$-terminal His ${ }_{6}$ tag) and ThnA4-His ${ }_{6}$ (C-terminal His ${ }_{6}$ tag) proteins were performed as previously described ${ }^{9}$ with the following modifications: cultures of $E$. coli NCM631 harboring the plasmid pIZ227 ${ }^{41}$ and either pMPO784, $\left(\right.$ thnA4-His 6 ), pMPO760, $\left(\right.$ thnA3-His 6 ) or pMPO785 (His - $^{-t h n Y}$ ), were grown in LB medium containing ampicillin and chloramphenicol overnight. They were used to inoculate 1 liter of Terrific Broth medium ${ }^{29}$ supplemented with extra iron and sulfur sources $(0.1 \mathrm{mg} / \mathrm{ml} \mathrm{ferric} \mathrm{ammo-}$ nium citrate, $0.1 \mathrm{mg} / \mathrm{ml}$ ferric citrate, $0.1 \mathrm{mg} / \mathrm{ml}$ iron sulfate heptahydrate, $0.1 \times$ ferrous sulfate/chelate solution, and $1 \mathrm{mM}$ cysteine), and $4 \mathrm{mg} / \mathrm{ml}$ riboflavin for $\mathrm{His}_{6}$-ThnY and ThnA4-His ${ }_{6}$. Expression of thnA3-His or $_{6}$ thnA4-His ${ }_{6}$ was induced by adding 0.5 or $0.05 \mathrm{mM}$ of isopropyl $\beta$-D-thiogalactopyranoside respectively, for $15 \mathrm{~h}$ at $16^{\circ} \mathrm{C}$. For ThnA4-His 6 , it was necessary to add $2 \mathrm{mM}$ FAD during the cell disruption by sonication to obtain a ferredoxin reductase with both, the iron sulfur-cluster and flavin, cofactors bound. Cell extracts were applied to an IMAC column (TALON ${ }^{\mathrm{TM}}$ resin (Clontech)) according to the manufacturer's specifications and bound proteins were eluted with an increasing imidazole gradient. Fractions exhibiting the expecting size and with color, indicative that some chromophores must be bound, were pooled, concentrated and applied to a Superdex $75 \mathrm{pg}$ 
column (10/60). Purified proteins were stored at $-80^{\circ} \mathrm{C}$ in $50 \mathrm{mM}$ Tris- $\mathrm{HCl}$ buffer, $\mathrm{pH} 7.4$ containing $250 \mathrm{mM}$ $\mathrm{NaCl}$, and $10 \%$ glycerol. To measure $\mathrm{His}_{6}-\mathrm{ThnY}$ and ThnA4-His ${ }_{6}$ concentrations, the extinction coefficient of each protein $\left(\varepsilon_{454 \mathrm{~nm}}=21.8 \mathrm{M}^{-1} \mathrm{~cm}^{-1}\right)$ and $\left(\varepsilon_{461 \mathrm{~nm}}=19.5 \mathrm{M}^{-1} \mathrm{~cm}^{-1}\right)$ respectively, were calculated using the same procedure described for ThnY-His ${ }_{6}{ }^{9}$. ThnA ${ }_{3}-\mathrm{His}_{6}$ concentration was determined by the Bradford method using bovine serum albumin as the standard. The application of different methods (including absorption spectroscopic methods and Fe quantification by inductively coupled plasma) in the attempt to determinate the protein extinction coefficient, indicated that [2Fe-2S] cluster incorporation was incomplete, with an average of $35 \%$ of incorporation. This prevented determination of the extinction coefficient. UV-Vis spectra were recorded either in a Shimadzu UVPC-1603 or in a Cary-100 spectrophotometer.

Stopped-flow pre-steady-state kinetic measurements. Transient electron transfer reactions among different couples of the Thn proteins, as well as from $\mathrm{NAD}(\mathrm{P}) \mathrm{H}$ to all of them were analyzed (reduction by $\mathrm{NAD}(\mathrm{P}) \mathrm{H}$ was only efficiently observed for ThnA4) by following the absorption spectral evolution in the flavin and iron sulfur regions (400-900 mm) using an Applied Photophysics SX17MV stopped-flow equipped with a photodiode array detector at $15^{\circ} \mathrm{C}^{30}$. All samples were prepared in potassium phosphate $50 \mathrm{mM}, \mathrm{pH} \mathrm{7.4,} \mathrm{NaCl}$ $10 \mathrm{mM}$, glycerine $5 \%$. Multiple wavelength absorption data were collected and processed using the X-Scan software (Appl. Phot. Ltd). Time spectral deconvolution was performed by global analysis and numerical integration methods using Pro-Kineticist (Appl. Phot. Ltd.). The collected data were fitted either to single-step, $\mathrm{A} \rightarrow \mathrm{B}$, two-steps, $\mathrm{A} \rightarrow \mathrm{B} \rightarrow \mathrm{C}$, three-steps, $\mathrm{A} \rightarrow \mathrm{B} \rightarrow \mathrm{C} \rightarrow \mathrm{D}$, or four-steps $\mathrm{A} \rightarrow \mathrm{B} \rightarrow \mathrm{C} \rightarrow \mathrm{D} \rightarrow \mathrm{E}$ models allowing estimation of the observed conversion rate constants $\left(k_{\mathrm{A} \rightarrow \mathrm{B}}, k_{\mathrm{B} \rightarrow \mathrm{C}}, k_{\mathrm{C} \rightarrow \mathrm{D}}\right)$. These observed rate constants describe the reaction under a particular set of conditions, being not limiting values, and are estimated with errors lower than $\pm 10 \%{ }^{31}$. However, values representing processes involving $\mathrm{ThnA}_{3}$ must be considered only from the qualitative point of view due to the low proportion of holoprotein exhibited by this protein. Model validity was assessed by lack of systematic deviations from residual plots at different wavelengths, inspection of calculated spectra and consistence among the number of significant singular values with the fit model. $k_{\mathrm{A} \rightarrow \mathrm{B}}$ rate constants derived from experimental data for the reaction between $\mathrm{NAD}(\mathrm{P}) \mathrm{H},\left(2.5-150 \mu \mathrm{M}\right.$ range), and ThnA $4_{\text {ox }}$ showed a dependence profile on the nucleotide concentration that fit to the equation describing binding at a single site followed by the electron transfer:

$$
k_{\mathrm{A} \rightarrow \mathrm{B}}=\frac{k_{\mathrm{red}}\left(K_{d}+[N A D(P) H]\right)+k_{\text {reox }}\left[N A D(P)^{+}\right]}{[N A D(P) H]+K_{d}}
$$

allowing to estimate limiting values at equilibrium for the dissociation $\left(K_{\mathrm{d}}\right)$ and the hydride transfer rate constants for the forward and reverse reactions $\left(k_{\text {red }}, k_{\text {reox }}\right)$ as previously described ${ }^{32,33}$. In general, errors in the estimated values for $K_{\mathrm{d}}$ and $k_{\text {red }}$ were lower than $\pm 20 \%$ and $\pm 15 \%$, respectively.

Determination of midpoint reduction potentials for ThnA4, ThnA3 and ThnY. Potentiometric titrations of ThnA4, ThnA 3 and ThnY, were attempted at $15^{\circ} \mathrm{C}$ by photoreduction under anaerobic conditions. Solutions contained $5 \mu \mathrm{M}$ ThnA4 or ThnY, or $20 \mu \mathrm{M}$ ThnA3, as well as $3 \mathrm{mM}$ EDTA and $2 \mu \mathrm{M} 5$-deazariboflavin. Measurements were carried out in potassium phosphate $50 \mathrm{mM}, \mathrm{pH} 7.4, \mathrm{NaCl} 10 \mathrm{mM}$, glycerine $5 \%$ for ThnA3 and ThnA4, and in HEPES 0.1 M pH 7.4, guanidine chloride $0.1 \mathrm{M}$, DTT $1 \mathrm{mM}$, EDTA $0.1 \mathrm{mM}$, glycerine $17 \%$ for ThnY. As mediators we used benzylviologen $\left(E_{\mathrm{m}}=-359 \mathrm{mV}\right)$ and indigo disulphonate $\left(E_{\mathrm{m}}=-125 \mathrm{mV}\right)$ for ThnA3; indigo disulphonate, benzylviologen and 1,2-naphtoquinone $\left(E_{\mathrm{m}}=+143 \mathrm{mV}\right)$ for ThnY, and benzylviologen, indigo disulphonate and anthraquinone-2-sulphonate $\left(E_{\mathrm{m}}=-225 \mathrm{mV}\right)$ for ThnA4. Stepwise reduction of the proteins was achieved by photoreduction and potentials along reduction were determined using a calomel electrode as reference $\left(E_{\mathrm{m}}=-251.1 \mathrm{mV}\right.$ at $\left.15^{\circ} \mathrm{C}\right)$ and a gold electrode as working one, as previously described ${ }^{34}$. The system was considered equilibrated when the potential of the solution $(E)$, measured with a Fluke 177 true-RMS multimeter, remained stable for at least $10 \mathrm{~min}$. UV-vis absorbance spectrum was then recorded. In the case of ThnA4, the extremely slow stabilization of the potential values after each reduction step as well as the subsequent denaturation of the protein upon reduction enforced us to measure potential values and their corresponding absorption spectra after 15 min of each illumination step. This was in detriment of the accuracy in determination of the midpoint potentials for this protein. Experiments were performed in duplicate.

Data were analyzed using the Origin Software (OriginLab) based on previously described methods ${ }^{35-39}$ using Equation 2 for ThnA3 and Equation 3 for ThnY and ThnA4. These equations, derived by extension to the Nernst equation and the Beer-Lambert Law, respectively describe a one-electron redox process or the sum of a one-electron process and a two-electron redox process;

$$
\begin{gathered}
\text { RelAbs }=\frac{\left(\mathrm{SFox} * 10^{(E-E \mathrm{mSF}) / 57}+\mathrm{SF}_{\mathrm{red}}\right)}{\left(1+10^{(\mathrm{E}-\mathrm{EmSF}) / 57}\right)} \\
\operatorname{RelAbs}=\frac{\left(\mathrm{FSox} * 10^{(E-E \mathrm{mSF}) / 57}+\mathrm{FS}_{\mathrm{red}}\right)}{\left(1+10^{(E-E \mathrm{mSF}) / 57}\right)}+\frac{\left(\mathrm{FADox} * 10^{(E-E \mathrm{mFAD}) / 28.5}+\mathrm{FAD}_{\mathrm{red}}\right)}{\left(1+10^{(E-E \mathrm{mFAD}) / 28.5}\right)}
\end{gathered}
$$

where RelAbs corresponds to the ratio between the sample absorption, at a given wavelength and at a particular stage of the reduction, and the maximal observed for such wavelength; $\mathrm{SFe}_{\mathrm{ox}}, \mathrm{SFe}_{\text {red }}, \mathrm{FAD}_{\mathrm{ox}}$, and $\mathrm{FAD}_{\text {red }}$ correspond to the relative contribution to the total RelAbs at each wavelength and stage of reduction of the oxidized and reduced forms of the $[2 \mathrm{Fe}-2 \mathrm{~S}]$ and FAD redox centers; $E$ is the experimentally measured potential at each state of the reduction; and $E_{\mathrm{mSFox} / \mathrm{red}}$ and $E_{\mathrm{mFADox} / \mathrm{red}}$ correspond respectively to the one-electron midpoint potential 
of the $[2 \mathrm{Fe}-2 \mathrm{~S}]$ cluster and the two-electron midpoint potential of the flavin cofactor. The complexity of the system, particularly for ThnY and ThnA4 (three-electron titration with probable overlap of midpoint potentials and the presence of several dyes) necessitated the use of a global fitting process. Thus RelAbs at several wavelengths were simultaneously plotted against the redox potential of the solution ( $\mathrm{mV} / \mathrm{SHE}$ ) and fit to either Eq. 2 or Eq. 3. Errors in the determined $E_{m \circ x}$ red were estimated to be $\pm 8 \mathrm{mV}$ for ThnA3 and ThnY, but values larger than $\pm 30 \mathrm{mV}$ are expected for ThnA4.

\section{References}

1. Mason, J. R. \& Cammack, R. The electron-transport proteins of hydroxylating bacterial dioxygenases. Annu. Rev. Microbiol. 46, 277-305 (1992).

2. Shingler, V. Integrated regulation in response to aromatic compounds: from signal sensing to attractive behaviour. Environ. Microbiol. 5, 1226-1241 (2003).

3. Andujar, E., Hernaez, M. J., Kaschabek, S. R., Reineke, W. \& Santero, E. Identification of an extradiol dioxygenase involved in tetralin biodegradation: gene sequence analysis and purification and characterization of the gene product. J. Bacteriol. 182, 789-795 (2000).

4. Hernaez, M. J. et al. Identification of a serine hydrolase which cleaves the alicyclic ring of tetralin. J. Bacteriol. 182, 5448-5453 (2000).

5. Hernaez, M. J., Floriano, B., Rios, J. J. \& Santero, E. Identification of a hydratase and a class II aldolase involved in biodegradation of the organic solvent tetralin. Appl. Environ. Microbiol. 68, 4841-4846 (2002).

6. Moreno-Ruiz, E., Hernaez, M. J., Martinez-Perez, O. \& Santero, E. Identification and functional characterization of Sphingomonas macrogolitabida strain TFA genes involved in the first two steps of the tetralin catabolic pathway. J. Bacteriol. 185, 2026-2030 (2003).

7. Lopez-Sanchez, A., Floriano, B., Andujar, E., Hernaez, M. J. \& Santero, E. Tetralin-induced and ThnR-regulated aldehyde dehydrogenase and beta-oxidation genes in Sphingomonas macrogolitabida strain TFA. Appl. Environ. Microbiol. 76, 110-118 (2010).

8. Lopez-Sanchez, A., Rivas-Marin, E., Martinez-Perez, O., Floriano, B. \& Santero, E. Co-ordinated regulation of two divergent promoters through higher-order complex formation by the LysR-type regulator ThnR. Mol. Microbiol. 73, 1086-1100 (2009).

9. Garcia, L. L. et al. ThnY is a ferredoxin reductase-like iron-sulfur flavoprotein that has evolved to function as a regulator of tetralin biodegradation gene expression. J. Biol. Chem. 286, 1709-1718 (2011).

10. Martinez-Perez, O., Lopez-Sanchez, A., Reyes-Ramirez, F., Floriano, B. \& Santero, E. Integrated response to inducers by communication between a catabolic pathway and its regulatory system. J. Bacteriol. 189, 3768-3775 (2007).

11. Ledesma-Garcia, L., Reyes-Ramirez, F. \& Santero, E. The ferredoxin ThnA3 negatively regulates tetralin biodegradation gene expression via ThnY, a ferredoxin reductase that functions as a regulator of the catabolic pathway. Plos One 8, e73910 (2013).

12. Nam, J. W. et al. Crystal structure of the ferredoxin component of carbazole 1,9a-dioxygenase of Pseudomonas resinovorans strain CA10, a novel Rieske non-heme iron oxygenase system. Proteins 58, 779-789 (2005).

13. Tucker, N. P. et al. The transcriptional repressor protein NsrR senses nitric oxide directly via a [2Fe-2S] cluster. Plos One 3, e3623 (2008).

14. Yamaguchi, M. \& Fujisawa, H. Purification and characterization of an oxygenase component in benzoate 1,2-dioxygenase system from Pseudomonas arvilla C-1. J. Biol. Chem. 255, 5058-5063 (1980).

15. Haigler, B. E. \& Gibson, D. T. Purification and properties of NADH-ferredoxinNAP reductase, a component of naphthalene dioxygenase from Pseudomonas sp. strain NCIB 9816. J. Bacteriol. 172, 457-464 (1990).

16. Fetzner, S., Muller, R. \& Lingens, F. Purification and some properties of 2-halobenzoate 1,2-dioxygenase, a two-component enzyme system from Pseudomonas cepacia 2CBS. J. Bacteriol. 174, 279-290 (1992).

17. Nam, J. W. et al. Purification and characterization of carbazole 1,9a-dioxygenase, a three-component dioxygenase system of Pseudomonas resinovorans strain CA10. Appl. Environ. Microbiol. 68, 5882-5890 (2002).

18. Gassner, G. T., Ludwig, M. L., Gatti, D. L., Correll, C. C. \& Ballou, D. P. Structure and mechanism of the iron-sulfur flavoprotein phthalate dioxygenase reductase. FASEB J. 9, 1411-1418 (1995).

19. Subramanian, V., Liu, T. N., Yeh, W. K., Narro, M. \& Gibson, D. T. Purification and properties of NADH-ferredoxinTOL reductase. A component of toluene dioxygenase from Pseudomonas putida. J. Biol. Chem. 256, 2723-2730 (1981).

20. Axcell, B. C. \& Geary, P. J. Purification and some properties of a soluble benzene-oxidizing system from a strain of Pseudomonas. Biochem. J. 146, 173-183 (1975).

21. Kimura, S., Kikuchi, A., Senda, T., Shiro, Y. \& Fukuda, M. Tolerance of the Rieske-type [2Fe-2S] cluster in recombinant ferredoxin BphA3 from Pseudomonas sp. KKS102 to histidine ligand mutations. Biochem. J. 388, 869-878 (2005).

22. Parsek, M. R., Shinabarger, D. L., Rothmel, R. K. \& Chakrabarty, A. M. Roles of CatR and cis,cis-muconate in activation of the catBC operon, which is involved in benzoate degradation in Pseudomonas putida. J. Bacteriol. 174, 7798-7806 (1992).

23. Schell, M. A. Transcriptional control of the nah and sal hydrocarbon-degradation operons by the nahR gene product. Gene 36, 301-309 (1985)

24. Mettert, E. L. \& Kiley, P. J. Fe-S proteins that regulate gene expression. Biochim. Biophys. Acta 1853, 1284-1293 (2015).

25. Colbert, C. L., Couture, M. M., Eltis, L. D. \& Bolin, J. T. A cluster exposed: structure of the Rieske ferredoxin from biphenyl dioxygenase and the redox properties of Rieske Fe-S proteins. Structure 8, 1267-1278 (2000).

26. Geary, P. J., Saboowalla, F., Patil, D. \& Cammack, R. An investigation of the iron-sulphur proteins of benzene dioxygenase from Pseudomonas putida by electron-spin-resonance spectroscopy. Biochem. J. 217, 667-673 (1984).

27. Couture, M. M. et al. Characterization of BphF, a Rieske-type ferredoxin with a low reduction potential. Biochemistry 40, 84-92 (2001).

28. Elsen, N. L., Moe, L. A., McMartin, L. A. \& Fox, B. G. Redox and functional analysis of the Rieske ferredoxin component of the toluene 4-monooxygenase. Biochemistry 46, 976-986 (2007).

29. Sambrook, J. \& Russell, D. W. Molecular cloning: A laboratory Manual, $3^{\text {rd }}$ Ed., Cold Spring Harbor Laboratory, Cold Spring Harbor, N.Y. (2001).

30. Lans, I. et al. Mechanism of the hydride transfer between Anabaena Tyr303Ser FNR(rd)/FNR(ox) and NADP +/H. A combined pre-steady-state kinetic/ensemble-averaged transition-state theory with multidimensional tunneling study. J. Phys. Chem. B 114, 3368-3379 (2010).

31. Tejero, J. et al. Catalytic mechanism of hydride transfer between NADP $+/ \mathrm{H}$ and ferredoxin-NADP+ reductase from Anabaena PCC 7119. Arch. Biochem. Biophys. 459, 79-90 (2007).

32. Sanchez-Azqueta, A. et al. A hydrogen bond network in the active site of Anabaena ferredoxin-NADP $(+)$ reductase modulates its catalytic efficiency. Biochim. Biophys. Acta 1837, 251-263 (2014).

33. Daff, S. An appraisal of multiple NADPH binding-site models proposed for cytochrome P450 reductase, NO synthase, and related diflavin reductase systems. Biochemistry 43, 3929-3932 (2004).

34. Frago, S. et al. Dual role of FMN in flavodoxin function: electron transfer cofactor and modulation of the protein-protein interaction surface. Biochim. Biophys. Acta 1797, 262-271 (2010).

35. Ravasio, S., Curti, B. \& Vanoni, M. A. Determination of the midpoint potential of the FAD and FMN flavin cofactors and of the 3 Fe4 S cluster of glutamate synthase. Biochemistry 40, 5533-5541 (2001). 
36. Roberts, G. A. et al. A self-sufficient cytochrome $\mathrm{p} 450$ with a primary structural organization that includes a flavin domain and a [2Fe-2S] redox center. J. Biol. Chem. 278, 48914-48920 (2003).

37. Das, A. \& Sligar, S. G. Modulation of the cytochrome P450 reductase redox potential by the phospholipid bilayer. Biochemistry 48, 12104-12112 (2009).

38. Munro, A. W., Noble, M. A., Robledo, L., Daff, S. N. \& Chapman, S. K. Determination of the redox properties of human NADPHcytochrome P450 reductase. Biochemistry 40, 1956-1963 (2001).

39. Daff, S. N. et al. Redox control of the catalytic cycle of flavocytochrome P-450 BM3. Biochemistry 36, 13816-13823 (1997).

40. Hanahan, D. Studies on transformation of Escherichia coli with plasmids. J. Mol. Biol. 166, 557-580 (1983).

41. Govantes, F., Molina-Lopez, J. A. \& Santero, E. Mechanism of coordinated synthesis of the antagonistic regulatory proteins NifL and NifA of Klebsiella pneumoniae. J. Bacteriol. 178, 6817-6823 (1996).

\section{Acknowledgements}

We wish to thank Guadalupe Martín for technical help. This work was supported by Spanish Ministry of Science and Innovation grants BIO2011-24003, CSD2007-00005, BIO2013-42978-P, and B-18 DGA-FEDER.

\section{Author Contributions}

L.L.-G. designed and performed the experiments with the help of A.S.-A. M.M. designed, supervised the biochemical work and analyzed data. F.R.-R. and E.S. conceived the study and its global design and coordination. M.M. and F.R.-R. wrote the main manuscript with the help of E.S. and L.L.-G. All authors read and approved the final manuscript.

\section{Additional Information}

Supplementary information accompanies this paper at http://www.nature.com/srep

Competing financial interests: The authors declare no competing financial interests.

How to cite this article: Ledesma-García, L. et al. Redox proteins of hydroxylating bacterial dioxygenases establish a regulatory cascade that prevents gratuitous induction of tetralin biodegradation genes. Sci. Rep. 6, 23848; doi: 10.1038/srep23848 (2016).

(c) (i) This work is licensed under a Creative Commons Attribution 4.0 International License. The images or other third party material in this article are included in the article's Creative Commons license, unless indicated otherwise in the credit line; if the material is not included under the Creative Commons license, users will need to obtain permission from the license holder to reproduce the material. To view a copy of this license, visit http://creativecommons.org/licenses/by/4.0/ 